\title{
Epidemic Profile of Maternal Syphilis in China in 2013
}

\author{
Lixia Dou, ${ }^{1}$ Xiaoyan Wang, ${ }^{1}$ Fang Wang, ${ }^{1}$ Qian Wang, ${ }^{1}$ Yaping Qiao, ${ }^{1}$ Min Su, ${ }^{1}$ Xi Jin, ${ }^{1}$ \\ Jie Qiu, ${ }^{2}$ Li Song, ${ }^{2}$ and Ailing Wang ${ }^{1}$ \\ ${ }^{1}$ National Center for Women and Children's Health, China CDC, No. 12 Dahuisi Road, Haidian District, Beijing 100081, China \\ ${ }^{2}$ National Health and Family Planning Commission, No. 14 Zhichun Road, Haidian District, Beijing 100088, China \\ Correspondence should be addressed to Li Song; songli@moh.gov.cn and Ailing Wang; ailing@chinawch.org.cn
}

Received 9 September 2015; Accepted 14 January 2016

Academic Editor: Kasonde Mwinga

Copyright (C) 2016 Lixia Dou et al. This is an open access article distributed under the Creative Commons Attribution License, which permits unrestricted use, distribution, and reproduction in any medium, provided the original work is properly cited.

\begin{abstract}
Objective. The aim of this study was to investigate the epidemiological characteristics and adverse pregnancy outcomes of pregnant women with syphilis infection in China. Methods. Data were from China's Information System of Prevention of Mother-to-Child Transmission of Syphilis Management. Women who were registered in the system and delivered in 2013 were included in the analysis. Results. A total of 15884 pregnant women with syphilis infection delivered in China in 2013. 79.1\% of infected women attended antenatal care at or before 37 gestational weeks; however, 55.4\% received no treatment or initiated the treatment after 37 gestational weeks. $14.0 \%$ of women suffered serious adverse pregnancy outcomes including stillbirth/neonatal death, preterm delivery/low birth weight, or congenital syphilis in newborns. High maternal titer $(\geq 1: 64)$ and late treatment $(>37$ gestational weeks)/nontreatment were significantly associated with increased risk of congenital syphilis and the adjusted ORs were 1.88 (95\% CI 1.27 to 2.80 ) and 3.70 (95\% CI 2.36 to 5.80), respectively. Conclusion. Syphilis affects a great number of pregnant women in China. Large proportions of women are not detected and treated at an early pregnancy stage. Burden of adverse pregnancy outcomes is high among infected women. Comprehensive interventions still need to be strengthened to improve uptake of screening and treatment for maternal syphilis.
\end{abstract}

\section{Introduction}

Pregnant women with syphilis infection can transmit the infection to their fetus and are at great risk for adverse pregnancy outcomes, such as miscarriage, stillbirth, preterm delivery, neonatal death, or congenital syphilis. Up to $80 \%$ of the women without effective treatment will experience these serious adverse pregnancy outcomes [1]. The harm caused by maternal and congenital syphilis among children may exceed that of HIV in developing countries [2]. Fortunately, adverse pregnancy outcomes including congenital syphilis can be effectively prevented by universal syphilis screening at an early pregnancy stage and treatment of those infected with penicillin, which can provide immediate benefits to both the infected mother and her fetus/infants. These interventions have been proved to be cost-effective, inexpensive, and feasible.

China has seen a dramatic resurgence of syphilis epidemic following the substantial changes in the society since mid1980s [3]. Along with the prevalence of syphilis among adults, congenital syphilis has become a public health problem in China and raised wide concern over its impact on children' health. It is reported that congenital syphilis has increased dramatically from 0.01 cases per 100000 live births in 1991 to 69.9 cases per 100000 live births in 2013, according to China's national sexually transmitted disease surveillance system [3, 4].

In recent years, China has endorsed a comprehensive strategy for the control of maternal syphilis and elimination of congenital syphilis. In 2011, China released the National Implementation Guidelines on Integrated Prevention of Mother-to-Child Transmission (iPMTCT) of HIV, syphilis, and HBV Programme. This programme is supported by government fund. According to the guidelines, cross-sectional collaboration and integrated approaches have been applied for maternal syphilis controlling and congenital syphilis elimination. Universal syphilis screening, counselling, and education are provided for all pregnant women, ideally at their first antenatal care visit. Either treponemal test or nontreponemal 
test will do for initial syphilis screening, though treponemal test is highly recommended. For those with positive syphilis tests appropriate treatment will be initiated promptly with at least 2 courses of penicillin treatment, one at early pregnancy stage and one at the third trimester. For each course women will receive either benzathine or procaine penicillin treatment by intramuscular injection, as recommended by the guidelines. Infants born to mothers with syphilis-infection will be followed up up to 18 months, until diagnosis of congenital syphilis is confirmed or excluded. Meanwhile, infants born to mother with inadequate treatment or no treatment will receive prophylactic benzathine penicillin treatment. Infants with confirmed congenital syphilis will be referred to specific health institutions for further treatment. In addition, the programme has established a maternal syphilis surveillance system, that is, China's Information System of Prevention of Mother-to-Child Transmission of Syphilis Management, in order to continuously monitor disease burden and trends of maternal and congenital syphilis in China.

However, maternal syphilis has been poorly investigated in China. There are only a few studies that reported the epidemic of maternal syphilis in local areas in China. Using nationwide surveillance data this paper aims to describe the epidemiological characteristics of pregnant women with maternal syphilis and their pregnancy outcomes in China in 2013. We also tried to identify the missed opportunities for maternal syphilis control and congenital syphilis prevention. These analyses will help raise the awareness of policy makers, researchers, and other stakeholders on this public health problem and improve intervention implementation on maternal and congenital syphilis control.

\section{Methods}

China's Information System of Prevention of Mother-toChild Transmission of Syphilis Management is a nationwide, health facility-based case report system. The system was established in 2011 and has been used to monitor and evaluating the prevalence of maternal syphilis and congenital syphilis in China. Surveillance of maternal syphilis is conducted through mandatory case-reporting by health facilities including general hospitals, maternal and children's hospitals, and other health providers across China, involving all 31 provinces, municipalities, and autonomous regions in the country. Diagnosis of maternal syphilis is based on at least one of the following situations in pregnant women: (1) positive treponemal and nontreponemal tests; (2) laboratory confirmation of Treponema pallidum in clinical specimens by dark-field microscopy; or (3) reactive treponemal antibody test of IgM. Pregnant women who met the diagnostic criteria are required to be registered in the system and followed up through pregnancy and postpartum period. Maternal syphilis case-reporting moved from paper reports to electronic online submission from 2013. Figure 1 shows the reporting structure of the surveillance system.

The surveillance system collected maternal syphilis data using standard forms. Relevant information on maternal syphilis was extracted by health staff from medical records.

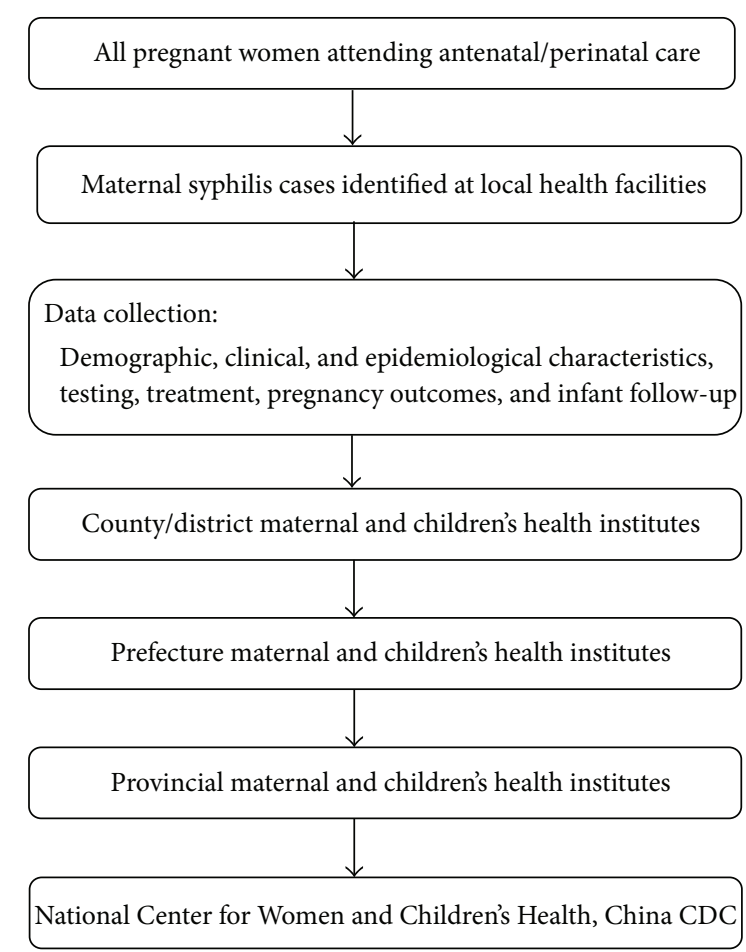

FIGURE 1: Reporting structure of China's Information System of Prevention of Mother-to-Child Transmission of Syphilis Management.

For each maternal syphilis case, data collected include information on demographic characteristics (e.g., maternal age, ethnicity, marital status, occupation, and education), maternal syphilis information (e.g., diagnostic evidence, stage of syphilis, route of acquisition, sexual partner's syphilis status, and laboratory testing results), and pregnancy outcomes and infant follow-up information (e.g., mode of delivery, preterm delivery, low birth weight, and congenital syphilis).

We used data extracted from the above surveillance system in this study. Pregnant women who met the following criteria and their children were included in the analysis: (1) syphilis screening tests positive during pregnancy and delivery at health facilities; (2) being diagnosed with syphilis; (3) being registered in the surveillance system; (4) delivered at gestational age of 28 weeks or more in 2013. Newborns with congenital syphilis born to women who received no syphilis tests were excluded from the analyses.

Migrating people were defined as those who lived in a different city/prefecture other than the place where their household was registered. The definition of syphilis stage is in accordance with the national guidelines [5]. In brief, primary syphilis is a stage of Treponema pallidum infection characterized by one or more chancres and demonstration of T. pallidum in clinical specimens by dark-field microscopy or reactive nontreponemal and treponemal tests. Secondary syphilis is characterized by maculopapular rash and often with lymphadenopathy and laboratory criteria as for primary syphilis. Tertiary syphilis is defined as a case with a history of primary, secondary, or latent syphilis that has clinical cardiovascular or central nervous system symptoms and signs 
and reactive nontreponemal tests or elevated cerebrospinal fluid protein or leukocyte count. Latent syphilis is defined as an asymptomatic case that has reactive nontreponemal and treponemal tests. Inadequate treatment for maternal syphilis was defined as nontreatment or nonpenicillin treatment, less than 2 completed courses of treatment during pregnancy, or less than 2 weeks between the 2 courses of treatment. Preterm delivery was defined as a delivery that occurs before 37 gestational weeks. Low birth weight (LBW) was defined as birth weight of a live-born baby of less than 2,500 $\mathrm{g}$ at birth. Stillbirth was defined as death of a fetus of at least 28 gestational weeks. Neonatal death was defined as death of live-born baby who died during the first week after birth. Congenital syphilis in newborn was defined as having at least one of the following situations in infant who was younger than 6 weeks and born to syphilis infected mother: (1) positive treponemal test and high titer of nontreponemal test (a value 4-fold higher than that of his/her mother's before delivery); (2) laboratory confirmation of Treponema pallidum in clinical specimens by dark-field microscopy; or (3) reactive treponemal antibody test of IgM. "Any adverse pregnancy outcomes" are a composite outcome in our analysis, which include preterm delivery, LBW, stillbirth, neonatal death, and congenital syphilis in newborns.

Statistical analysis was performed using SPSS 20.0. Values for categorical variables were presented as numbers and percentages. Values for continuous variables were presented as means and standard deviations (SD). Comparisons were made between regions of eastern, central, and western China [6] using $\chi^{2}$ statistic for categorical variables and $F$ statistic (analysis of variances) for continuous variables. $P$ values $<$ 0.05 were considered of statistical significance. Crude ORs (cOR) and adjusted ORs (aOR) were calculated using logistic regression to explore potential factors associated with congenital syphilis.

\section{Results}

3.1. Overall Characteristics of the Population. In 2013, a total of 15884 pregnant women with laboratory-confirmed syphilis infection delivered and were registered in Information System of Prevention of Mother-to-Child Transmission of Syphilis Management in China. Proportions of infected women from eastern, central, and western China were $41.7 \%$, $23.9 \%$, and $34.4 \%$, respectively. Table 1 shows the demographic characteristics of these infected pregnant women. The majority $(79.5 \%)$ of the women were aged between 20 and 34 years old. In eastern and central China, women of minority ethnicity accounted for less than $10 \%$, while in the western area nearly $40 \%$ were minority. $23.4 \%$ of infected women were migrating people. $6 \%$ were unmarried (including those being single, cohabitated, divorced, or widowed) during the index pregnancy. $41.1 \%$ were housewives or unemployed, ranging from $25.9 \%$ in western area to $52.5 \%$ in eastern area. $79.1 \%$ of infected women attended antenatal care at or before 37 gestational weeks, while $16.8 \%$ attended antenatal care after 37 gestational weeks and $4.1 \%$ were unknown.
3.2. Clinical Characteristics of Pregnant Women with Syphilis Infection. Table 2 shows the syphilis characteristics of infected pregnant women in China in 2013. 18.3\% of women reported that they had had syphilis infection before the index pregnancy. Proportions of women with latent syphilis, primary syphilis, secondary syphilis, and tertiary syphilis were $67.6 \%, 6.2 \%, 1.0 \%$, and $0.5 \%$, respectively, leaving $24.7 \%$ of those with syphilis stage being unknown. Only $55.6 \%$ of infected women had their syphilis detected during pregnancy, and more than $40 \%$ remained undetected until delivery or postpartum period. Proportions of women who initiated the treatment for maternal syphilis during the first and second trimesters were $12.4 \%$ and $19.8 \%$, respectively. $55.4 \%$ were untreated or initiated the treatment after 37 gestational weeks. Nearly $60 \%$ of women received penicillin treatment for maternal syphilis, while $37.9 \%$ received no treatment at all. $68.8 \%$ of infected women's husband/sexual partners did not undertake any syphilis tests and their infection status was unknown during the entire period of the index pregnancy. Among those who received syphilis tests, $26.3 \%$ were reported to test positive for syphilis.

\subsection{Pregnancy Outcomes and Complications of Pregnant} Women with Syphilis Infection. Table 3 shows the pregnancy outcomes of pregnancy women with syphilis infection in China in 2013. The mean gestational age at delivery was 39.2 weeks. $51.9 \%$ of women gave birth by vaginal delivery and $46.3 \%$ by caesarean section. $14.0 \%$ of women suffered adverse pregnancy outcomes, and proportions of women who experienced stillbirth/neonatal death, preterm delivery/low birth weight, and neonatal congenital syphilis were $2.9 \%$, $10.5 \%$, and $3.0 \%$, respectively.

3.4. Associations between Clinical Characteristics of Syphilis Infected Pregnant Women and Congenital Syphilis in Newborns. Table 4 shows that in both univariable and multivariable models, maternal titer of nontreponema and gestational week for the first treatment were statistically associated with congenital syphilis in newborns. Women with high maternal titer $(\geq 1: 64)$ had greater risk of having babies with congenital syphilis than those with low titer $(\leq 1: 4)$ (adjusted OR (aOR) 1.88, 95\% CI 1.27 to 2.80 ). Compared with early treatment for infection ( $\leq 14$ gestational weeks), treatment initiated during 29-37 weeks and late treatment ( $>37$ gestational weeks)/nontreatment were significantly associated with increased risk of congenital syphilis (aOR 3.20, 95\% CI 1.94 to 5.27 , and aOR $3.70,95 \%$ CI 2.36 to 5.80 , resp.).

\section{Discussion}

Data in this study came from China's Information System of Prevention of Mother-to-Child Transmission of Syphilis Management. Identified maternal syphilis cases are mandatory to be registered to the system in China. The established system can provide useful evidence for policy-makers and other stakeholders on planning, implementing, and evaluating public health policies on maternal and congenital syphilis control. In our study, only pregnant women with 
TABLE 1: Demographic characteristics of pregnant women with syphilis infection in China in 2013, by region ${ }^{\mathrm{a}}$.

\begin{tabular}{|c|c|c|c|c|c|}
\hline \multirow{2}{*}{ Characteristic } & \multirow{2}{*}{ Total } & \multicolumn{3}{|c|}{ Region } & \multirow{2}{*}{$P$ value } \\
\hline & & Eastern & Central & Western & \\
\hline \multicolumn{6}{|l|}{ Maternal age (years) } \\
\hline$<20$ & $1138(7.2 \%)$ & 437 (6.6\%) & $221(5.8 \%)$ & $480(8.8 \%)$ & \multirow{3}{*}{$<0.001$} \\
\hline $20-34$ & $12627(79.5 \%)$ & $5310(80.2 \%)$ & $3072(80.9 \%)$ & $4245(77.6 \%)$ & \\
\hline$\geq 35$ & $2119(13.3 \%)$ & $872(13.2 \%)$ & $505(13.3 \%)$ & $742(13.6 \%)$ & \\
\hline \multicolumn{6}{|l|}{ Ethnicity } \\
\hline Han & $13100(82.5 \%)$ & $6229(94.1 \%)$ & $3558(93.7 \%)$ & $3313(60.6 \%)$ & \multirow{2}{*}{$<0.001$} \\
\hline Minority & $2784(17.5 \%)$ & $390(5.9 \%)$ & $240(6.3 \%)$ & $2154(39.4 \%)$ & \\
\hline \multicolumn{6}{|l|}{ Residency status } \\
\hline Local resident & $12170(76.6 \%)$ & $4233(64.0 \%)$ & $3304(87.0 \%)$ & $4633(84.7 \%)$ & \multirow{2}{*}{$<0.001$} \\
\hline Migrating people & $3714(23.4 \%)$ & $2386(36.0 \%)$ & $494(13.0 \%)$ & $834(15.3 \%)$ & \\
\hline \multicolumn{6}{|l|}{ Marital status } \\
\hline Single & $643(4.0 \%)$ & $406(6.1 \%)$ & $95(2.5 \%)$ & $142(2.6 \%)$ & \multirow{5}{*}{$<0.001$} \\
\hline First married & $13658(86.0 \%)$ & $5662(85.5 \%)$ & $3339(87.9 \%)$ & $4657(85.2 \%)$ & \\
\hline Remarried & $1267(8.0 \%)$ & $397(6.0 \%)$ & $302(8.0 \%)$ & $568(10.4 \%)$ & \\
\hline Cohabitated & $235(1.5 \%)$ & $105(1.6 \%)$ & $47(1.2 \%)$ & $83(1.5 \%)$ & \\
\hline Divorced/widowed & $81(0.5 \%)$ & $49(0.7 \%)$ & $15(0.4 \%)$ & $17(0.3 \%)$ & \\
\hline \multicolumn{6}{|l|}{ Education } \\
\hline Primary or lower & $2982(18.8 \%)$ & $955(14.4 \%)$ & $469(12.3 \%)$ & $1558(28.5 \%)$ & \multirow{5}{*}{$<0.001$} \\
\hline Junior middle school & $8290(52.2 \%)$ & $3313(50.1 \%)$ & $2166(57.0 \%)$ & $2811(51.4 \%)$ & \\
\hline Senior middle school & $2921(18.4 \%)$ & $1441(21.8 \%)$ & $743(19.6 \%)$ & $737(13.5 \%)$ & \\
\hline College or above & $896(5.6 \%)$ & $502(7.6 \%)$ & $191(5.0 \%)$ & $203(3.7 \%)$ & \\
\hline Unknown & $795(5.0 \%)$ & $408(6.2 \%)$ & $229(6.0 \%)$ & $158(2.9 \%)$ & \\
\hline \multicolumn{6}{|l|}{ Occupation } \\
\hline Farmers & $5828(36.7 \%)$ & $1315(19.9 \%)$ & $1294(34.1 \%)$ & $3219(58.9 \%)$ & \multirow{3}{*}{$<0.001$} \\
\hline Housewife/unemployed & $6521(41.1 \%)$ & $3477(52.5 \%)$ & $1630(42.9 \%)$ & $1414(25.9 \%)$ & \\
\hline Others & $3535(22.3 \%)$ & $1827(27.6 \%)$ & $874(23.0 \%)$ & $834(15.3 \%)$ & \\
\hline \multicolumn{6}{|l|}{ Gravidity } \\
\hline 1 & $4065(25.6 \%)$ & $1571(23.7 \%)$ & $1200(31.6 \%)$ & $1294(23.7 \%)$ & \multirow{3}{*}{$<0.001$} \\
\hline $2-3$ & 8637 (54.4\%) & $3656(55.2 \%)$ & $1953(51.4 \%)$ & $3028(55.4 \%)$ & \\
\hline$>3$ & $3182(20.0 \%)$ & $1392(21.0 \%)$ & $645(17.0 \%)$ & $1145(20.9 \%)$ & \\
\hline \multicolumn{6}{|l|}{ Parity } \\
\hline 0 & $5289(33.3 \%)$ & $2302(34.8 \%)$ & $1331(35.0 \%)$ & $1656(30.3 \%)$ & \multirow{3}{*}{$<0.001$} \\
\hline $1-2$ & $9778(61.6 \%)$ & $4013(60.6 \%)$ & $2331(61.4 \%)$ & $3434(62.8 \%)$ & \\
\hline$>3$ & 817 (5.1\%) & $304(4.6 \%)$ & $136(3.6 \%)$ & 377 (6.9\%) & \\
\hline \multicolumn{6}{|c|}{ First antenatal care (gestational weeks) } \\
\hline$\leq 14$ & $6379(40.2 \%)$ & $2556(38.6 \%)$ & $1687(44.4 \%)$ & $2136(39.1 \%)$ & \multirow{5}{*}{$<0.001$} \\
\hline $15-28$ & $4175(26.3 \%)$ & $2003(30.3 \%)$ & $824(21.7 \%)$ & $1348(24.7 \%)$ & \\
\hline $29-37$ & $2007(12.6 \%)$ & $849(12.8 \%)$ & $431(11.3 \%)$ & $727(13.3 \%)$ & \\
\hline$>37$ & $2665(16.8 \%)$ & $861(13.0 \%)$ & $718(18.9 \%)$ & $1086(19.9 \%)$ & \\
\hline Unknown & $658(4.1 \%)$ & $350(5.3 \%)$ & $138(3.6 \%)$ & $170(3.1 \%)$ & \\
\hline
\end{tabular}

${ }^{\mathrm{a}}$ Values are given as number (percentage) unless otherwise indicated.

syphilis infection that delivered in 2013 were eligible for inclusion. Hence, a total of 15884 women were included in the analysis. We analyzed the demographic, clinical, and syphilis related characteristics of these infected pregnant women in China, as well as their pregnancy outcomes. Our findings indicate that most pregnant women with syphilis infection received penicillin treatment; however, there were still large proportions of women that had not been detected and treated at an early pregnancy stage. Vast intervention opportunities were missed to prevent adverse pregnancy outcomes caused by maternal syphilis.

China commenced the integrated programme of prevention of mother-to-child transmission (iPMTCT) of human immunodeficiency virus (HIV), syphilis, and hepatitis B virus (HBV) in 2010. Free HIV, syphilis, and HBV tests are provided to all pregnant women. In 2013, the syphilis 
TABLE 2: Clinical characteristics of pregnant women with syphilis infection in China in 2013, by region ${ }^{\mathrm{a}}$.

\begin{tabular}{|c|c|c|c|c|c|}
\hline \multirow{2}{*}{ Characteristic } & \multirow{2}{*}{ Total } & \multicolumn{3}{|c|}{ Region } & \multirow{2}{*}{$P$ value } \\
\hline & & Eastern & Central & Western & \\
\hline \multicolumn{6}{|c|}{ History of syphilis infection } \\
\hline Yes & $2910(18.3 \%)$ & $1537(23.2 \%)$ & $566(14.9 \%)$ & $807(14.8 \%)$ & \multirow{2}{*}{$<0.001$} \\
\hline No & $12974(81.7 \%)$ & $5082(76.8 \%)$ & $3232(85.1 \%)$ & $4660(85.2 \%)$ & \\
\hline \multicolumn{6}{|l|}{ Maternal syphilis stage } \\
\hline Latent syphilis & $10742(67.6 \%)$ & $4751(71.8 \%)$ & $2193(57.7 \%)$ & $3798(69.5 \%)$ & \multirow{5}{*}{$<0.001$} \\
\hline Primary syphilis & $981(6.2 \%)$ & $421(6.4 \%)$ & $234(6.2 \%)$ & $326(6.0 \%)$ & \\
\hline Secondary syphilis & $158(1.0 \%)$ & $81(1.2 \%)$ & $48(1.3 \%)$ & $29(0.5 \%)$ & \\
\hline Tertiary syphilis & $73(0.5 \%)$ & $39(0.6 \%)$ & $17(0.4 \%)$ & $17(0.3 \%)$ & \\
\hline Unknown & $3930(24.7 \%)$ & $1327(20.0 \%)$ & $1306(34.4 \%)$ & $1297(23.7 \%)$ & \\
\hline \multicolumn{6}{|c|}{ Period of maternal syphilis detection } \\
\hline During pregnancy & $8829(55.6 \%)$ & $4136(62.5 \%)$ & $1728(45.5 \%)$ & $2965(54.2 \%)$ & \multirow{4}{*}{$<0.001$} \\
\hline At delivery & $5246(33.0 \%)$ & $1675(25.3 \%)$ & $1619(42.6 \%)$ & $1952(35.7 \%)$ & \\
\hline Postpartum & $1722(10.8 \%)$ & $771(11.6 \%)$ & $430(11.3 \%)$ & $521(9.5 \%)$ & \\
\hline Other & $87(0.5 \%)$ & $37(0.6 \%)$ & $21(0.6 \%)$ & $29(0.5 \%)$ & \\
\hline \multicolumn{6}{|c|}{ Maternal titer of nontreponema } \\
\hline$\leq 1: 4$ & $11118(70.0 \%)$ & $4780(72.2 \%)$ & $2587(68.1 \%)$ & $3751(68.6 \%)$ & \multirow{4}{*}{$<0.001$} \\
\hline $1: 8-1: 32$ & $3736(23.5 \%)$ & $1405(21.2 \%)$ & $1006(26.5 \%)$ & $1325(24.2 \%)$ & \\
\hline$\geq 1: 64$ & $565(3.6 \%)$ & $261(3.9 \%)$ & $111(2.9 \%)$ & $193(3.5 \%)$ & \\
\hline Unknown & $465(2.9 \%)$ & $173(2.6 \%)$ & $94(2.5 \%)$ & $198(3.6 \%)$ & \\
\hline \multicolumn{6}{|c|}{ Gestational week for the first treatment } \\
\hline$\leq 14$ & $1972(12.4 \%)$ & $1033(15.6 \%)$ & $297(7.8 \%)$ & $642(11.7 \%)$ & \multirow{4}{*}{$<0.001$} \\
\hline $15-28$ & $3151(19.8 \%)$ & $1498(22.6 \%)$ & $500(13.2 \%)$ & $1153(21.1 \%)$ & \\
\hline $29-37$ & $1966(12.4 \%)$ & $815(12.3 \%)$ & $388(10.2 \%)$ & $763(14.0 \%)$ & \\
\hline$>37$ or untreated & $8795(55.4 \%)$ & $3273(49.4 \%)$ & $2613(68.8 \%)$ & $2909(53.2 \%)$ & \\
\hline \multicolumn{6}{|l|}{ Drug of treatment } \\
\hline Penicillin & $9386(59.1 \%)$ & $3940(59.5 \%)$ & $1785(47.0 \%)$ & $3661(67.0 \%)$ & \multirow{3}{*}{$<0.001$} \\
\hline Nonpenicillin & $485(3.1 \%)$ & $253(3.8 \%)$ & $99(2.6 \%)$ & $133(2.4 \%)$ & \\
\hline Untreated & $6013(37.9 \%)$ & $2426(36.7 \%)$ & $1914(50.4 \%)$ & $1673(30.6 \%)$ & \\
\hline \multicolumn{6}{|c|}{ Syphilis testing of husband/sexual partner } \\
\hline Negative & $3654(23.0 \%)$ & $1616(24.4 \%)$ & $890(23.4 \%)$ & $1148(21.0 \%)$ & \multirow{3}{*}{$<0.001$} \\
\hline Positive & $1302(8.2 \%)$ & $542(8.2 \%)$ & $275(7.2 \%)$ & $485(8.9 \%)$ & \\
\hline Untested/unknown & $10928(68.8 \%)$ & $4461(67.4 \%)$ & $2633(69.3 \%)$ & $3834(70.1 \%)$ & \\
\hline
\end{tabular}

${ }^{\mathrm{a}}$ Values are given as number (percentage) unless otherwise indicated.

testing coverage in pregnant women was 96.40\% [7]. All syphilis infected women will be provided free intramuscular penicillin treatment.

Migrating women, women with lower education level, and women who are jobless or housewives/farmers are usually socioeconomically disadvantaged. In our study, we found that this less advantaged population accounted for a large proportion of maternal syphilis cases in China. Former studies showed that prevalence of maternal syphilis in migrating population was higher than that in local residents and their compliance to treatment tended to be poor [8]. Extra attention and commitment should be made to promote the uptake of syphilis tests and compliance of treatment in this vulnerable population.

China's iPMTCT guidelines recommend that all pregnant women should be subjected to a syphilis screening either with nontreponemal tests or treponemal tests at their first antenatal care visit. Early prenatal care is an important component for prevention of congenital syphilis because it can facilitate early detection of maternal syphilis and prompt treatment for those with positive tests $[9,10]$. It was estimated that one week's delay in initiation of antenatal care could approximately raise the risk of congenital syphilis by $10 \%$ [11]. Our study showed that delayed or absence of antenatal care was not uncommon among women with syphilis infection. Only $40 \%$ initiated antenatal care during the first trimester. Around $56 \%$ of women with maternal syphilis were identified during pregnancy and more than $40 \%$ were diagnosed at delivery or postpartum period. Several reasons are probably behind this worrying situation of low recognition of the disease. First, screening tests for syphilis might be unavailable temporally in some clinics because test kits were out of stock; second, health providers might have not asked women to undertake the tests; third, but not the least, women 
TABle 3: Pregnancy outcomes of women with syphilis infection in China in 2013, by region ${ }^{\text {a }}$.

\begin{tabular}{|c|c|c|c|c|c|}
\hline \multirow{2}{*}{ Characteristic } & \multirow{2}{*}{ Total } & \multicolumn{3}{|c|}{ Region } & \multirow{2}{*}{$P$ value } \\
\hline & & Eastern & Central & Western & \\
\hline Gestational weeks at delivery (mean \pm SD) & $39.2 \pm 2.2$ & $39.1 \pm 2.3$ & $39.2 \pm 2.3$ & $39.3 \pm 2.1$ & $<0.001$ \\
\hline \multicolumn{6}{|l|}{ Mode of delivery } \\
\hline Spontaneous VD ${ }^{\mathrm{b}}$ & $7900(49.7 \%)$ & $3388(51.2 \%)$ & $1473(38.8 \%)$ & $3039(55.6 \%)$ & \multirow{5}{*}{$<0.001$} \\
\hline Instrumental $\mathrm{VD}^{\mathrm{b}}$ & $351(2.2 \%)$ & $107(1.6 \%)$ & $102(2.7 \%)$ & $142(2.6 \%)$ & \\
\hline Elective $\mathrm{CS}^{\mathrm{c}}$ & $4355(27.4 \%)$ & $1821(27.5 \%)$ & $1390(36.6 \%)$ & $1144(20.9 \%)$ & \\
\hline Emergency $\mathrm{CS}^{\mathrm{c}}$ & $3005(18.9 \%)$ & $1055(15.9 \%)$ & $820(21.6 \%)$ & $1130(20.7 \%)$ & \\
\hline Unknown & $273(1.7 \%)$ & $248(3.7 \%)$ & $13(0.3 \%)$ & $12(0.2 \%)$ & \\
\hline \multicolumn{6}{|l|}{ Stillbirth/neonatal death } \\
\hline Yes & $454(2.9 \%)$ & $200(3.0 \%)$ & $121(3.2 \%)$ & $133(2.4 \%)$ & \multirow{2}{*}{0.059} \\
\hline No & $15430(97.1 \%)$ & $6419(97.0 \%)$ & $3677(96.8 \%)$ & $5334(97.6 \%)$ & \\
\hline \multicolumn{6}{|l|}{ Preterm delivery/low birth weight } \\
\hline Yes & $1665(10.5 \%)$ & $728(11.0 \%)$ & $408(10.7 \%)$ & $529(9.7 \%)$ & \multirow{2}{*}{0.051} \\
\hline No & $14219(89.5 \%)$ & $5891(89.0 \%)$ & $3390(89.3 \%)$ & $4938(90.3 \%)$ & \\
\hline \multicolumn{6}{|l|}{ Neonatal congenital syphilis } \\
\hline Yes & $478(3.0 \%)$ & $191(2.9 \%)$ & $181(4.8 \%)$ & $106(1.9 \%)$ & \multirow{2}{*}{$<0.001$} \\
\hline No & $15406(97.0 \%)$ & $6428(97.1 \%)$ & $3617(95.2 \%)$ & $5361(98.1 \%)$ & \\
\hline \multicolumn{6}{|l|}{ Any adverse pregnancy outcomes } \\
\hline Yes & $2223(14.0 \%)$ & $946(14.3 \%)$ & $598(15.7 \%)$ & $679(12.4 \%)$ & \multirow{2}{*}{$<0.001$} \\
\hline No & $13661(86.0 \%)$ & $5673(85.7 \%)$ & $3200(84.3 \%)$ & $4788(87.6 \%)$ & \\
\hline
\end{tabular}

${ }^{\mathrm{a}}$ Values are given as number (percentage) or mean $\pm \mathrm{SD}$ unless otherwise indicated; ${ }^{\mathrm{b}} \mathrm{VD}$ refers to vaginal delivery; ${ }^{\mathrm{c}} \mathrm{SD}$ refers to caesarean section.

TABLE 4: Logistic regression analysis of syphilis infected pregnant women's clinical characteristics and congenital syphilis in newborns.

\begin{tabular}{|c|c|c|c|c|c|}
\hline & \multirow{2}{*}{$n(\%)$} & \multicolumn{2}{|c|}{ Univariable } & \multicolumn{2}{|c|}{ Multivariable $^{\mathrm{a}}$} \\
\hline & & $\mathrm{cOR}^{\mathrm{b}}(95 \% \mathrm{CI})$ & $P$ Value & $\mathrm{aOR}^{\mathrm{c}}(95 \% \mathrm{CI})$ & $P$ Value \\
\hline \multicolumn{6}{|c|}{ History of syphilis infection } \\
\hline Yes & $74(2.5 \%)$ & - & & - & \\
\hline No & $404(3.1 \%)$ & $1.23(0.96,1.58)$ & 0.104 & $0.98(0.76,1.27)$ & 0.870 \\
\hline \multicolumn{6}{|l|}{ Maternal syphilis stage } \\
\hline Latent syphilis & $275(2.6 \%)$ & - & & - & \\
\hline Primary syphilis & $38(3.9 \%)$ & $1.53(1.09,2.17)$ & 0.015 & $1.38(0.97,1.96)$ & 0.071 \\
\hline Secondary syphilis & $8(5.1 \%)$ & $2.03(0.99,4.18)$ & 0.054 & 1. $58(0.76,3.27)$ & 0.220 \\
\hline Tertiary syphilis & $0(0.0 \%)$ & NA & & NA & \\
\hline Unknown & $157(4.0 \%)$ & $1.58(1.30,1.93)$ & $<0.001$ & $1.42(1.15,1.74)$ & 0.001 \\
\hline \multicolumn{6}{|c|}{ Maternal titer of nontreponema } \\
\hline$\leq 1: 4$ & $279(2.5 \%)$ & - & & - & \\
\hline $1: 8-1: 32$ & $150(4.0 \%)$ & $1.63(1.33,1.99)$ & $<0.001$ & $1.60(1.30,1.97)$ & $<0.001$ \\
\hline$\geq 1: 64$ & $29(5.1 \%)$ & $2.10(1.42,3.11)$ & $<0.001$ & $1.90(1.28,2.83)$ & 0.002 \\
\hline Unknown & $20(4.3 \%)$ & $1.75(1.10,2.78)$ & 0.018 & $1.37(0.86,2.19)$ & 0.189 \\
\hline \multicolumn{6}{|c|}{ Gestational week for the first treatment } \\
\hline$\leq 14$ & $21(1.1 \%)$ & - & & - & \\
\hline $15-28$ & $49(1.6 \%)$ & $1.47(0.88,2.45)$ & 0.144 & $1.41(0.84,2.37)$ & 0.192 \\
\hline $29-37$ & $67(3.4 \%)$ & $3.28(2.00,5.37)$ & $<0.001$ & $3.20(1.94,5.27)$ & $<0.001$ \\
\hline$\geq 37$ or untreated & $341(3.9 \%)$ & $3.75(2.41,5.84)$ & $<0.001$ & $3.70(2.36,5.80)$ & $<0.001$ \\
\hline
\end{tabular}

${ }^{a}$ The model was adjusted for maternal age, residence location, education, and job.

${ }^{b}$ cOR refers to crude odds ratio.

${ }^{c}$ aOR refers to adjusted odds ratio. 
with syphilis infection might be reluctant to take the tests either because of worries about stigma, costs, or beliefs of unnecessary. All sexual partners of pregnant women should undertake syphilis tests and those with positive test should receive treatment, as recommended by China's iPMTCT guidelines. We found that syphilis tests were performed for about $30 \%$ of sexual partners of pregnant women with syphilis infection. For nearly $70 \%$ of the partners the syphilis infection status was unknown. This could put women at high risk of reinfection of the disease, even after effective treatment. To address these issues, testing and counselling services should be further strengthened at antenatal clinics.

Penicillin treatment is necessary to improve health outcomes for both women and children [12, 13]. China's iPMTCT guidelines recommend that all pregnant women with syphilis infection should receive adequate treatment to prevent congenital syphilis. In 2013, less than a third of women with syphilis infection initiated treatment before the third trimester, while more than a third received no treatment at all. Hence, a large proportion of pregnant women were untreated or inadequately treated, which posed great risk for congenital syphilis. Patient education, along with free treatment services, should be improved to increase women's awareness of the infection and their willingness for treatment. In addition, undocumented treatment needs to be investigated to get better understanding of the treatment situation. Our study showed that $14 \%$ of women suffered serious adverse pregnancy outcomes, including stillbirth/neonatal death $(10.5 \%)$, preterm delivery/low birth weight $(2.9 \%)$, or neonatal congenital syphilis (3.0\%). Previous studies in China showed that in general population the prevalence rates of stillbirth and preterm delivery were around $0.3 \%$ [14] and $3.8 \%$ [15], respectively, which is much lower than those with syphilis infection. Infected women were still at great risk for adverse pregnancy outcomes and maternal syphilis continues to be an important cause for morbidity and mortality in pregnancy. However, adverse pregnancy outcomes caused by maternal syphilis can be almost entirely averted by early detection and prompt treatment [16]. The high prevalence of adverse pregnancy outcomes highlighted the gaps existing in current antenatal cares.

In 2007, World Health Organization (WHO) called on global elimination of congenital syphilis by 2015 and developed strategies to combat the disease, including strong political will, improved access to antenatal care, screening and treatment, and surveillance system [17]. In response, China has shown great political will to achieve this goal by introducing universal maternal syphilis screening and treatment for positive cases, along with other interventions, such as improving access, uptake, and quality of early prenatal care, providing education and counseling, and sexual partner testing and treatment if necessary. Currently, high antenatal care attendance level, cost-effective diagnostic testing, and the widespread availability of penicillin are already in place across the country. But maternal and congenital syphilis continue being a serious public health problem and elimination of congenital syphilis is still a big challenge to China. Barriers that hamper the uptake of syphilis testing and treatment during pregnancy still exist and need to be identified and tackled. More commitment should be taken to enhance the implementation of maternal and congenital syphilis controlling programme.

This study has several limitations. First, our data were from China's Information System of Prevention of Motherto-Child Transmission of Syphilis Management, which is a passive surveillance system. It might be biased by low incidence reporting due to underdetection, misclassification, and underreporting, since data collection on maternal syphilis relies on health facility reporting. Second, only women who delivered at a gestational age of no less than 28 weeks were included in the analysis. Hence, we might underestimate the situation of maternal syphilis in China, for many may have miscarriages and fetal loss in the early stage of pregnancy. Third, only neonatal congenital syphilis cases rather than all congenital syphilis cases were included in our study. Because many infected infants are asymptomatic at birth or with subtle and nonspecific symptoms and maternal nontreponemal and treponemal antibodies can cross the placenta, this makes the diagnosis of congenital syphilis problematic. So infants born to mother with syphilis infection need to be evaluated with series of serological tests and followed up up to 18 months before diagnosis of congenital syphilis can be made or be excluded. However, by the time of our analysis, these data on all congenital syphilis cases were not available and were not analyzed in this paper.

According to our knowledge, this is the first study regarding the country's epidemic profile of maternal syphilis in China. Through our analysis on antenatal screening, maternal syphilis treatment, and pregnancy outcomes using the national data, we can see that the maternal syphilis picture in China is alarming. Integrated interventions on maternal syphilis still need to be further strengthened to promote maternal and children's health. Government should commit more to investment in infrastructure of public health, health provider capacity building, and population education. In addition, more studies are needed to explore barriers and strategies to elimination of congenital syphilis in China.

\section{Conclusions}

Syphilis continues to affect a large number of pregnant women in China. Universal maternal syphilis screening and treatment for positive cases have been in place for several years across the country, yet there were still large proportions of women that had not been detected and treated at an early pregnancy stage. Burden of adverse pregnancy outcomes is high among infected women. Barriers that hamper the uptake of syphilis testing and treatment during pregnancy still exist and need to be identified and tackled. China's integrated strategies for maternal syphilis control need to be further strengthened to improve access to antenatal care, syphilis screening, and treatment during early pregnancy.

\section{Conflict of Interests}

The authors declare that there is no conflict of interests regarding the publication of this paper. $b$ 


\section{References}

[1] H. Saloojee, S. Velaphi, Y. Goga, N. Afadapa, R. Steen, and O. Lincetto, "The prevention and management of congenital syphilis: an overview and recommendations," Bulletin of the World Health Organization, vol. 82, no. 6, pp. 424-430, 2004.

[2] World Health Organization, Guidelines for the Management of Sexually Transmitted Infections, World Health Organization, Geneva, Switzerland, 2003.

[3] Z.-Q. Chen, G.-C. Zhang, X.-D. Gong et al., "Syphilis in China: results of a national surveillance programme," The Lancet, vol. 369, no. 9556, pp. 132-138, 2007.

[4] X. D. Gong, X. L. Yue, F. Teng, N. Jiang, and P. X. Men, "Syphilis in China from 2000 to 2013: epidemiological trends and characteristics," Chinese Journal of Dermatology, vol. 47, no. 5, pp. 310-315, 2014.

[5] SBTS/MOH, "National standard of the People's Republic of China: diagnostic criteria and management of syphilis," GB 15974-1995, Standards Press of China, Beijing, China, 1996.

[6] National Health and Family Planning Commission of China, China Health Statistics Yearbook 2013, Peking Union Medical College Press, Beijing, China, 2014.

[7] A.-L. Wang, Y.-P. Qiao, L.-H. Wang et al., "Integrated prevention of mother-to-child transmission for human immunodeficiency virus, syphilis and hepatitis B virus in China," Bulletin of the World Health Organization, vol. 93, no. 1, pp. 52-56, 2015.

[8] L. P. Zhu, M. Qin, L. Du, R.-H. Xie, T. Wong, and S. W. Wen, "Maternal and congenital syphilis in Shanghai, China, 2002 to 2006," International Journal of Infectious Diseases, vol. 14, no. 3, pp. e45-e48, 2010.

[9] E. G. Lago, L. C. Rodrigues, R. M. Fiori, and A. T. Stein, "Congenital syphilis: identification of two distinct profiles of maternal characteristics associated with risk," Sexually Transmitted Diseases, vol. 31, no. 1, pp. 33-37, 2004.

[10] K. L. Southwick, S. Blanco, A. Santander et al., "Maternal and congenital syphilis in Bolivia, 1996: prevalence and risk factors," Bulletin of the World Health Organization, vol. 79, no. 1, pp. 3342, 2001.

[11] J.-B. Liu, F.-C. Hong, P. Pan et al., "A risk model for congenital syphilis in infants born to mothers with syphilis treated in gestation: a prospective cohort study," Sexually Transmitted Infections, vol. 86, no. 4, pp. 292-296, 2010.

[12] K. A. Workowski and S. Berman, "Sexually transmitted diseases treatment guidelines, 2010," MMWR Recommendations and Reports, vol. 59, no. RR12, pp. 1-110, 2010.

[13] J. M. Douglas, "Penicillin treatment of syphilis: clearing away the shadow on the land," The Journal of the American Medical Association, vol. 301, no. 7, pp. 769-771, 2009.

[14] L. Zhang, H. Li, and K. B. Liu, "Analysis of stillbirth prevalence in Beijing from 2010 to 2014," Beijing Medical Journal, vol. 37, no. 7, pp. 641-643, 2015.

[15] L. Liu, J. M. Liu, Y. H. Liu et al., "Prevalence of preterm birth among singletons in 10 counties (cities) of China, 1993-2005," Chinese Journal of Epidemiology, vol. 28, no. 11, pp. 1051-1054, 2007.

[16] World Health Organization and United Nations Children's Fund, Antenatal Care in Developing Countries: Promises, Achievements and Missed Opportunities: An Analysis of Trends, Levels and Differentials, 1990-2001, World Health Organization, United Nations Children's Fund, Geneva, Switzerland, 2003.
[17] World Health Organization, The Global Elimination of Congenital Syphilis: Rationale and Strategy for Action, World Health Organization, Geneva, Switzerland, 2007. 


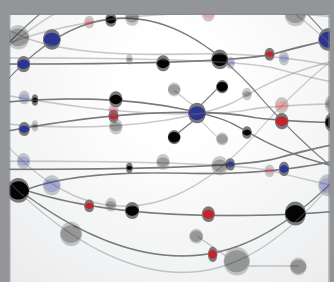

The Scientific World Journal
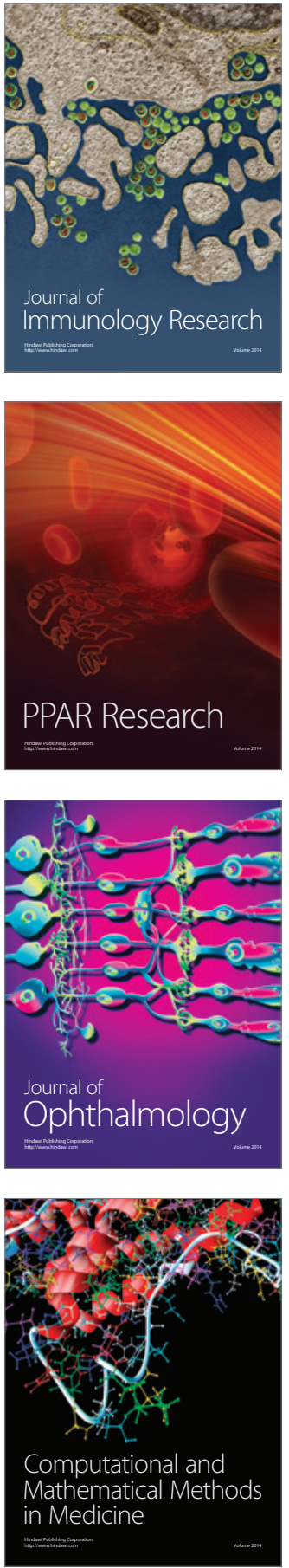

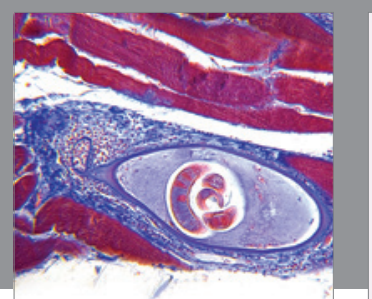

Gastroenterology Research and Practice

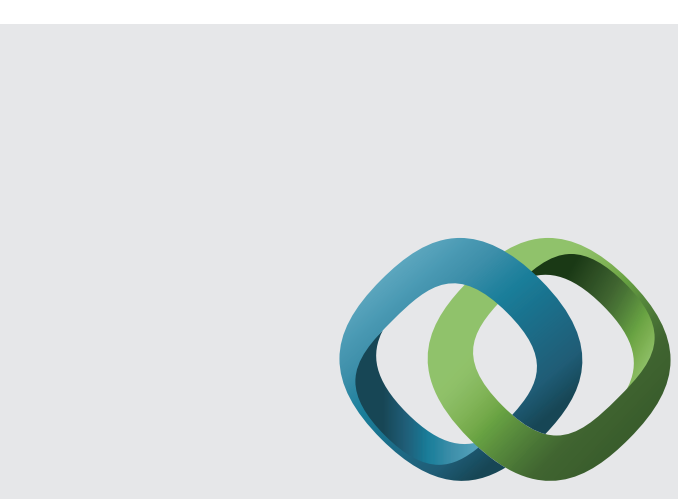

\section{Hindawi}

Submit your manuscripts at

http://www.hindawi.com
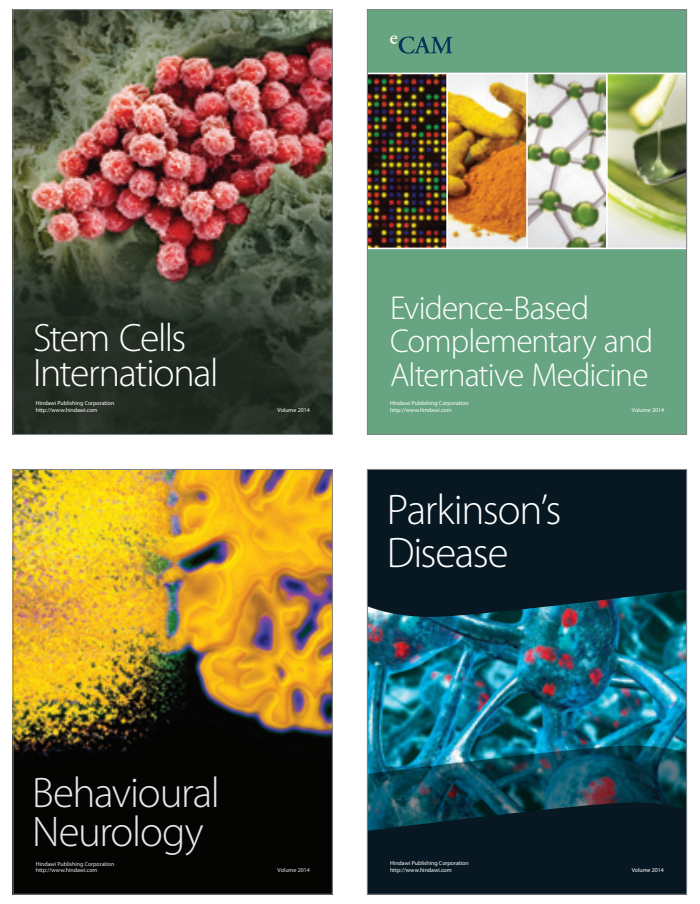
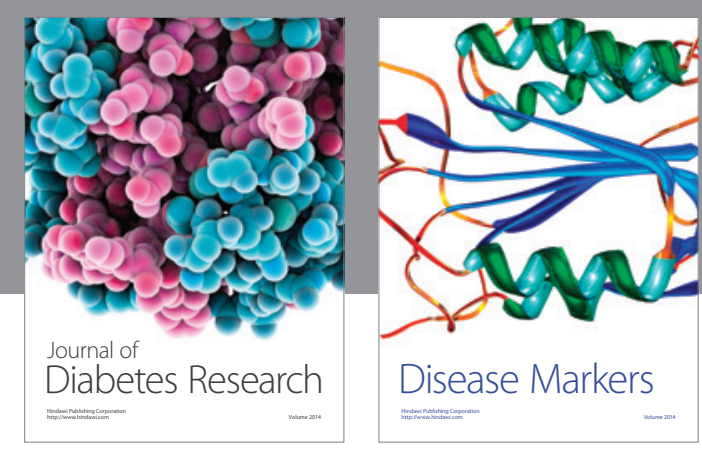

Disease Markers
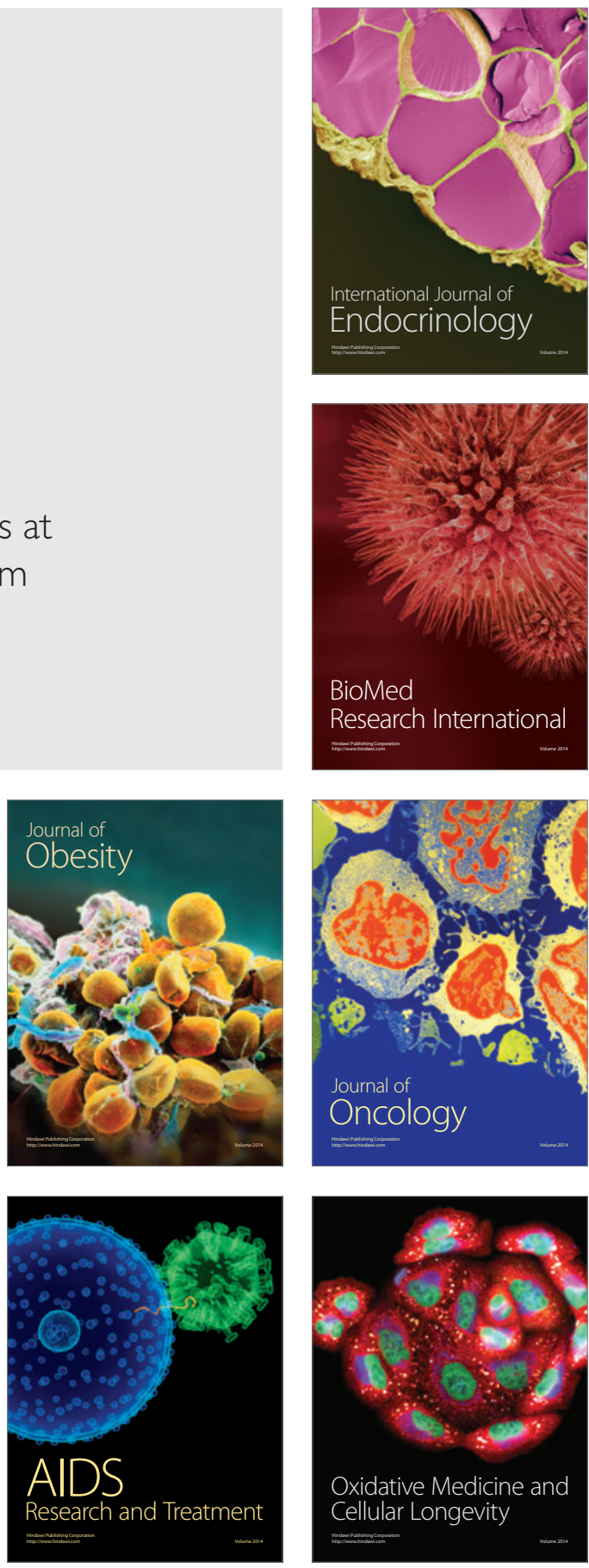